\title{
DIETITIANS
}

Laura Swaffield describes how dietitians can help a wide range of people through adapting what they eat.
USEFUL INFORMATION

\section{British Dietetic \\ Association}

Elizabeth House

22 Suffolk Street

Queensway

Birmingham B1 1LS

Tel: 01216435483
S tate registered dietitians have taken a degree in nutrition and dietetics. Their job is to use their scientific and medical

knowledge, and translate it into terms of real food. They advise people on what to eat, and devise special diets when needed.

Their advice needs to be tailored to your individual tastes, culture, lifestyle and budget. Otherwise you cannot be expected to follow it. So do not be shy about making comments and asking questions if you are discussing diet.

In hospitals, dietitians work closely with doctors, nurses and individual patients and also advise on catering. In the community, the work varies from giving catering advice (to schools or meals on wheels services, for instance), to health education campaigns, from giving talks to groups, to work with individuals.

\section{DIETARY NEEDS}

All kinds of people (including carers) might need to eat a diet different from the average. Long-term problems include cystic fibrosis, diabetes, kidney disease, irritable bowel syndrome, heart disease, allergies, and 'inborn errors of metabolism', which make common foods dangerous for some people to eat. Diet can also make a big difference in cancer, convalescence after any illness or surgery, and even wound healing.

If you or the person you care for has a medical condition that needs a special diet, you should be referred automatically to a state registered dietitian.
A better diet will also improve general health. It can also maintain good health after major illnesses, such as heart attacks. A high fibre diet can relieve constipation. And avoiding weight gain or losing weight often helps people with disabilities (and their carers!) to cope better.

Some people have problems in chewing or swallowing. This may be a temporary problem (for example in illness, or just after a stroke) or a lifelong one (sometimes found in disabled or mentally handicapped people, for instance). Dietitians can help ensure you eat well despite these problems.

Dietitians usually start by assessing how well nourished a person is, by asking about their normal diet and sometimes by having tests done. They will make sure that the information they give is suited to you and the person you care for and will help you understand and adapt your diet.

\section{TO CONTACT A DIETITIAN}

If you need dietary advice for a medical condition, you have to be referred by a doctor. This should happen automatically if you use any medical service (at a school, at a hospital or with a GP, for example).

For general advice, ask your GP if a community dietitian works at the practice (many do now). If not, try the chief or senior dietitian at the hospital as a first contact.

For more advice, contact the British Dietetic Association, which will also supply, on request, a list of local state registered dietitians working outside the NHS. Please send an s.a.e. 